\title{
Altered cardiovascular function at birth in growth-restricted preterm lambs
}

\author{
Graeme R. Polglase ${ }^{1,2}$, Beth J. Allison 1,2, Elise Coia ${ }^{1}$, Anqi Li', Graham Jenkin ${ }^{1,2}$, Atul Malhotra ${ }^{1,3}$, Arvind Sehgal ${ }^{1,3}$, \\ Martin Kluckow ${ }^{4}$, Andrew W. Gill'5, Stuart B. Hooper ${ }^{1,2}$ and Suzanne L. Miller ${ }^{1,2}$
}

BACKGROUND: Cardiovascular dysfunction at birth may underlie poor outcomes after fetal growth restriction (FGR) in neonates. We compared the cardiovascular transition between FGR and appropriately grown (AG) preterm lambs and examined possible mechanisms underlying any cardiovascular dysfunction in FGR lambs.

METHODS: FGR was induced in ewes bearing twins at 0.7 gestation; the twin was used as an internal control (AG). At 0.8 gestation, lambs were delivered and either euthanized with their arteries isolated for in vitro wire myography, or ventilated for $2 \mathrm{~h}$. At $60 \mathrm{~min}$, inhaled nitric oxide (iNO) was administered in a subgroup for $30 \mathrm{~min}$. Molecular assessment of the nitric oxide (NO) pathway within lung tissue was conducted.

RESULTS: FGR lambs had lower left ventricular output and cerebral blood flow (CBF) and higher systemic vascular resistance compared with AG lambs. INO administration to FGR lambs rapidly improved cardiovascular and systemic hemodynamics but resulted in decreased CBF in AG lambs. Isolated arteries from FGR lambs showed impaired sensitivity to NO donors, but enhanced vasodilation to Sildenafil and Sodium nitroprusside, and altered expression of components of the NO pathway.

CONCLUSION: Cardiovascular dysfunction at birth may underlie the increased morbidity and mortality observed in preterm FGR newborns. Impaired NO signaling likely underlies the abnormal vascular reactivity.

$\mathbf{F}^{\mathrm{e}}$ etal growth restriction (FGR) increases perinatal mortality and morbidity (1). Moderate to severe FGR occurs in $\sim 6 \%$ of pregnancies and is diagnosed by reduced fetal growth and deficits in placental blood flow (2,3). FGR infants require antenatal surveillance and intervention (e.g., premature delivery) to prevent fetal mortality, the incidence of which is from 10 to 20 -fold greater than in infants appropriately grown (AG) for their gestational age (1). Increased risk of perinatal mortality and morbidity is present irrespective of gestational age at delivery (4), and is associated with neonatal and ongoing complications, including respiratory distress, chronic lung disease, cardiovascular disease, and neurodevelopmental impairments $(1,5)$. However, the etiology of poor neonatal outcomes in FGR infants is not well understood.

Placental insufficiency is the leading cause of FGR, resulting in chronic fetal hypoxia. The fetus adapts to a low oxygen environment by altering cardiovascular output, redistributing blood flow to the brain and heart, to optimize oxygen and nutrient supply in an attempt to preserve normal function and growth in these vital organs (6). This cardiovascular adaptation is achieved by vasoconstriction in nonvital vascular beds, and results in characteristic asymmetric fetal growth restriction, with sparing of head and brain growth at the expense of musculoskeletal dimensions, and reduced fetal body growth and fat deposition (3). While this adaptive response ensures survival in utero, it may adversely affect fetal cardiovascular development (both heart and vasculature), which in turn, may significantly impact cardiovascular function during the transition to newborn life. Indeed, recent studies indicate that this fetal adaptive response to placental insufficiency is responsible for the unique pathophysiology of the FGR newborn, characterized by reduced cardiac output, hypotension and peripheral vasoconstriction evident within the first few days after birth $(7,8)$. We contend that these pathophysiological consequences likely underlie the complications and progression of many of the morbidities faced by preterm FGR infants, particularly evident during the cardiopulmonary hemodynamic transition at birth.

A successful cardiopulmonary transition from fetal to newborn life requires removal of lung liquid and commencement of pulmonary gas exchange, concomitant with functional reorganization of the cardiovascular system $(9,10)$. Lung aeration induces a rapid decrease in pulmonary vascular resistance and increase in pulmonary blood flow (PBF) to ensure efficient gas exchange, and thereby enabling PBF to become the sole source of left ventricular preload $(11,12)$. Failure of appropriate cardiovascular transition results in increased requirement for neonatal intensive care.

\footnotetext{
The last two authors contributed equally to this work.

'The Ritchie Centre, Hudson Institute of Medical Research, Clayton, Victoria, Australia; ${ }^{2}$ Department of Obstetrics and Gynecology, Monash University, Clayton, Victoria,

Australia; ${ }^{3}$ Monash Newborn, Monash Medical Centre, and Department of Pediatrics, Monash University, Melbourne, Victoria, Australia; ${ }^{4}$ Department of Neonatology, Royal North Shore Hospital and University of Sydney, Sydney, New South Wales, Australia; ${ }^{5}$ Centre for Neonatal Research and Education, The University of Western Australia, Perth, Western Australia, Australia. Correspondence: Graeme Polglase (graeme.polglase@monash.edu)

Received 28 February 2016; accepted 01 March 2016; advance online publication 29 June 2016. doi:10.1038/pr.2016.104
} 
This study aimed (i) to determine whether FGR causes cardiovascular and vascular dysfunction in preterm lambs; (ii) to determine whether fetal cardiovascular adaptations to placental insufficiency results in an impaired cardiovascular transition at preterm delivery; and (iii) to determine whether altered nitric oxide (NO) signaling is a potential mechanism of cardiovascular dysfunction. We utilized our established model of FGR, induced via single umbilical artery ligation (SUAL), which induces placental insufficiency, chronic hypoxia, and asymmetric growth restriction, thereby mimicking the

Table 1. Fetal and baseline characteristics

\begin{tabular}{lcccc}
\hline & $\mathrm{AG}_{\mathrm{UvC}}$ & $\mathrm{FGR}_{\mathrm{UVC}}$ & $\mathrm{AG}_{\text {vent }}$ & $\mathrm{FGR}_{\text {vent }}$ \\
\hline$N$ & 5 & 4 & 9 & 9 \\
Male $n,(\%)$ & $2(40)$ & $2(50)$ & $4(44)$ & $4(44)$ \\
$\mathrm{GA}(\mathrm{d})$ & $125.0 \pm 0.6$ & $125.0 \pm 0.6$ & $125.6 \pm 0.4$ & $125.6 \pm 0.4$ \\
Body weight $(\mathrm{kg})$ & $3.5 \pm 0.1$ & $2.5 \pm 0.2^{*}$ & $3.3 \pm 0.2$ & $2.3 \pm 0.1^{*}$ \\
Brain (g/kg) & $13.8 \pm 0.5$ & $17.7 \pm 1.1^{*}$ & $14.0 \pm 0.7$ & $20.5 \pm 1.3^{*}$ \\
Lungs (g/kg) & $37.3 \pm 5.1$ & $34.3 \pm 0.7$ & $35.2 \pm 1.2$ & $36.5 \pm 3.2$ \\
Heart (g/kg) & $7.6 \pm 0.4$ & $7.2 \pm 0.2$ & $7.1 \pm 0.3$ & $7.4 \pm 0.3$ \\
Mean fetal pH & $7.37 \pm 0.01$ & $7.37 \pm 0.01$ & $7.36 \pm 0.01$ & $7.34 \pm 0.01$ \\
Mean fetal PaO 2 & $22.6 \pm 1.3$ & $19.8 \pm 0.8^{*}$ & $24.1 \pm 0.3$ & $20.2 \pm 0.4^{*}$ \\
Mean fetal PaCO & $47.9 \pm 1.8$ & $48.6 \pm 1.7$ & $44.1 \pm 1.3$ & $46.2 \pm 0.7$ \\
Mean fetal SaO & $63.6 \pm 4.0$ & $59.1 \pm 2.4^{*}$ & $67.7 \pm 1.5$ & $56.4 \pm 0.9^{*}$ \\
\hline
\end{tabular}

AG, appropriately grown; FGR, fetal growth restriction; UVC, unventilated controls; Vent, ventilated lambs; $n$, number; GA; gestational age.

* Indicates significant difference $(P<0.05)$ FGR vs. AG. common clinical characteristics of human FGR (13-15). We hypothesized that FGR alters pulmonary, systemic, and cerebral vascular development, resulting in a poor cardiovascular transition at birth. We further hypothesized that alterations to NO signaling underlies poor cardiovascular dysfunction in preterm FGR newborn lambs.

\section{RESULTS}

\section{Baseline Characteristics and Physiological Parameters}

Baseline characteristics and mean fetal blood-gas data is detailed in Table 1. FGR lambs had significantly lower fetal $\mathrm{SaO}_{2}$ and $\mathrm{PaO}_{2}$ than $\mathrm{AG}$ preterm lambs, but had similar $\mathrm{pH}$ and $\mathrm{PaCO}_{2}$. FGR lambs were significantly smaller than AG lambs, but brain weight/body weight ratio was significantly increased in FGR lambs, indicative of brain sparing. FGR had no effect on lung or heart weights when corrected for bodyweight.

\section{Ventilations and Oxygenation Parameters}

Ventilation requirements, including peak inspiratory pressure, tidal volume, mean airway pressure (Figure 1a), and fraction of inspired oxygen were not different between groups at any time during ventilation. Respiratory system resistance, compliance, and ventilator efficiency index were not different between groups (data not shown). The partial pressure of oxygen, carbon dioxide (Figure 1b), and the alveolar-arterial difference in oxygen (Figure 1c) were not different between groups throughout ventilation. Cerebral tissue oxygenation tend to be $7.2 \pm 3 \%$ lower (mean (SEM)) in FGR compared with AG preterm lambs $(P=0.061$; Figure 1d).

\section{b}
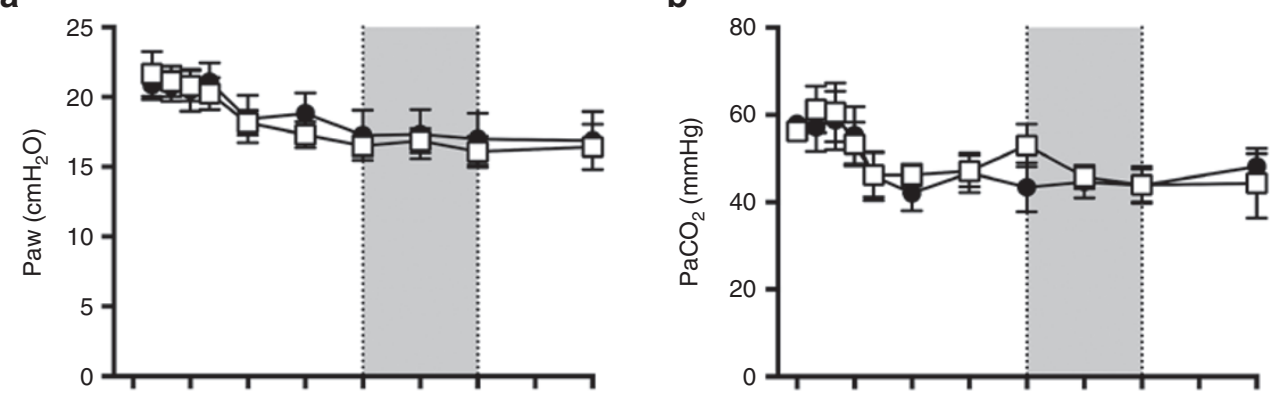

C

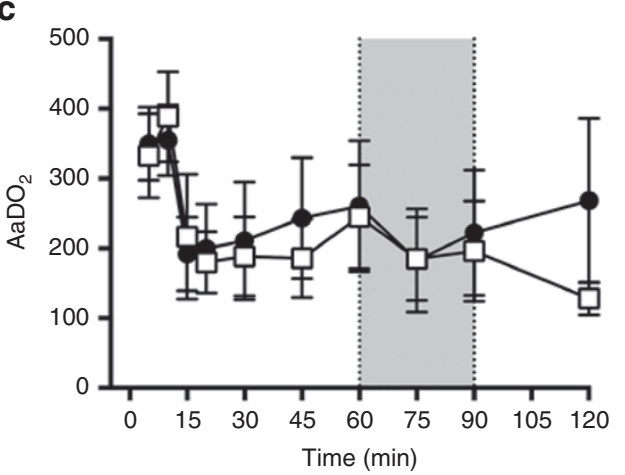

d

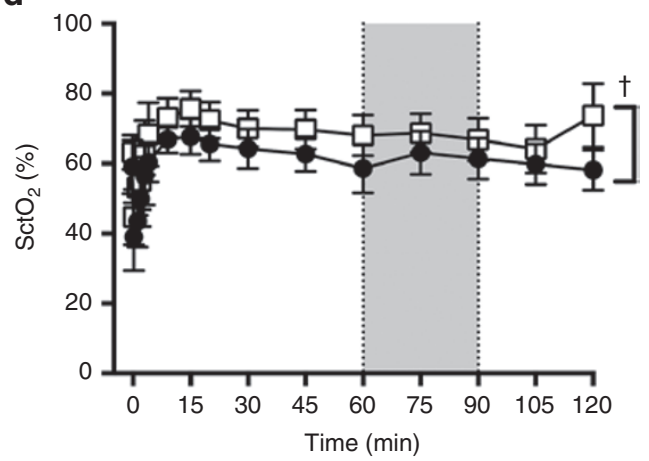

Figure 1. Respiratory overview. (a) Airway pressure (Paw), (b) Partial arterial pressure of carbon dioxide $\left(\mathrm{PaCO}_{2}\right)$, (c) alveolar-arterial difference in oxygen $\left(\mathrm{AaDO}_{2}\right.$ ), and (d) cerebral tissue oxygenation index (SctO2; measured by NIRS) in fetal growth restriction (FGR) (black circles) and appropriately grown (AG) (open squares) preterm lambs. Shaded region indicates time of inhaled nitric oxide (iNO) challenge. No differences in respiratory outcomes was

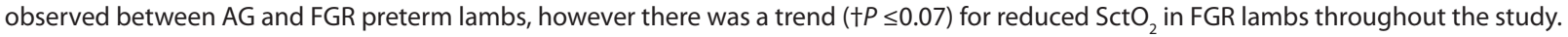




\section{Articles | Polglase et al.}

\section{Fetal Hemodynamics}

Immediately prior to delivery, mean fetal PBF was not different between groups, however peak systolic PBF was lower and end-diastolic PBF was higher in FGR preterm lambs indicative of reduced PBF amplitude (Figures 2a-c). Mean fetal CBF was lower in FGR compared with AG preterm lambs (Figure 2d). Fetal blood pressure and systemic arterial pulsatility index measured in the carotid artery were not different between groups.

\section{Transitional Hemodynamics}

After initiation of ventilation, mean PBF was not different between groups (Figure 2b). However, mean CBF was significantly lower in FGR lambs throughout the first 60 min of ventilation. Peak systolic CBF was not different, but FGR lambs had significantly lower end-diastolic CBF from $10 \mathrm{~min} ; 100 \%$ of FGR lambs and $66 \%$ of AG lambs had negative end diastolic $\mathrm{CBF}$, indicating ductal steal (Figure 2f). Blood pressure was not different between groups during the initial ventilation (Figure 2g). Systemic arterial pulsatility index was significantly higher in FGR lambs compared with AG lambs from
$20 \mathrm{~min}$, indicative of higher downstream vascular resistance (Figure 2h). All lambs rapidly developed a pure left to right shunt across the ductus arteriosus.

Four of nine FGR lambs had evidence of harmonic mean pulse pressure variability, with concurrent fluctuations in cerebral blood flow indicative of pressure passivity (Figure 3 ). This was not observed in AG preterm lambs.

Left ventricular output was significantly lower in FGR preterm lambs compared with AG preterm lambs, even when corrected for weight (Figure $2 \mathbf{i}$ ).

\section{Isolated Blood Vessel Assessment}

Given the findings of higher systemic vascular resistance in FGR lambs compared with AG lambs, we isolated blood vessels from femoral and middle-cerebral arteries from an additional group of FGR and AG preterm lambs to determine whether any functional differences were present using in vitro wire myography.

There was no difference in the contractile properties of isolated femoral or middle-cerebral arteries (as measured by high $\mathrm{K}+$ physiological saline solution and phenylephrine (femoral) a

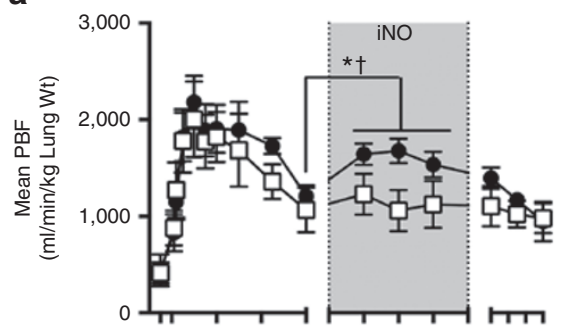

d

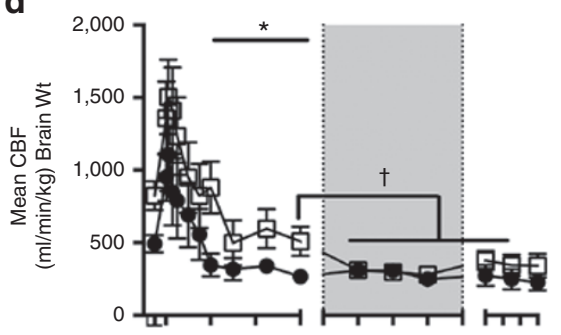

g

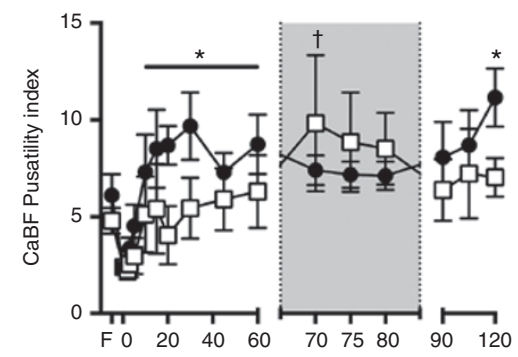

b

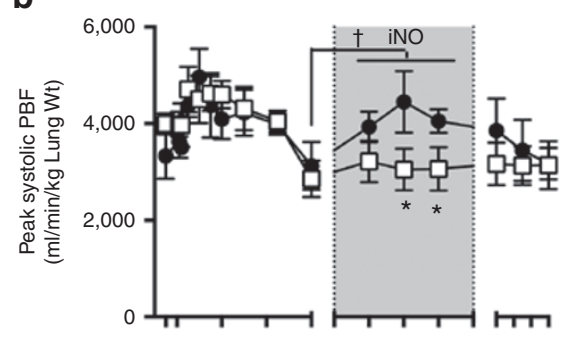

e

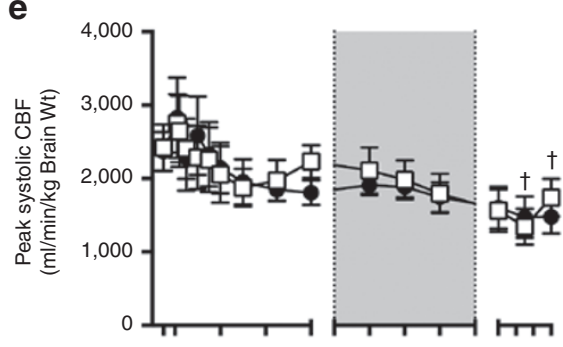

h

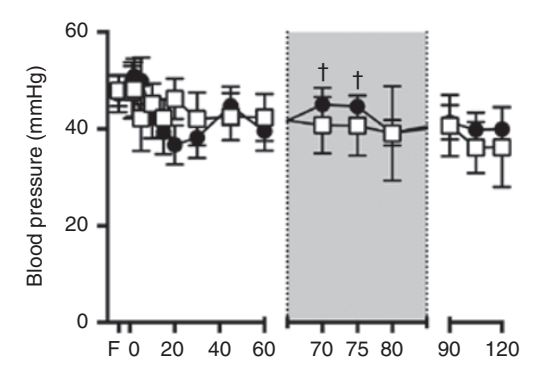

C

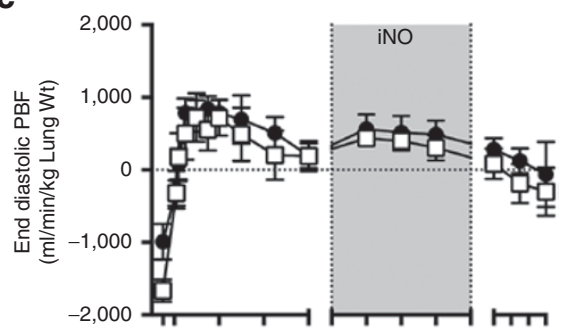

f

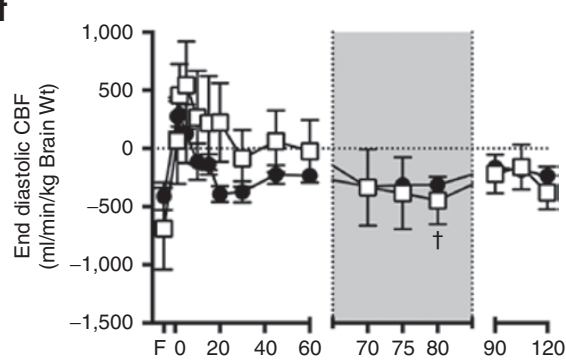

i

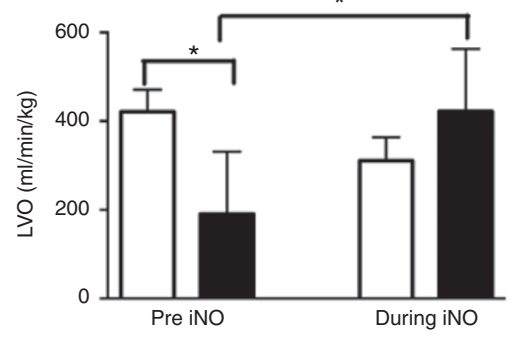

Figure 2. Cardiopulmonary transition at birth. (a) Mean, (b) peak systolic, and (c) end diastolic pulmonary blood flow (PBF) per gram of lung weight; (d) mean (e), peak systolic, and (f) end diastolic carotid blood flow (CBF) per gram of brain weight; (g) pulsatility index measured in the carotid artery and (h) blood pressure in fetal growth restriction (FGR) (black circles) and appropriately grown (AG) (open squares) preterm lambs. Measurements were taken 5 min prior to delivery (fetal: $F$ ) and regularly after initiation of ventilation and subsequent inhaled nitric oxide (iNO) administration. The area highlighted in gray indicates the period when iNO was administered, between 60 and 90 min after delivery. (i) Left ventricular output (LVO) measured using Doppler Echocardiography immediately prior to, and during iNO administration in FGR (black bars) and AG (open bars) preterm lambs. ${ }^{*}$ indicates significant difference $(P<0.05)$ between FGR and AG preterm lambs. ${ }^{\dagger}$ indicates significant time effect after iNO administration, determined by a significant difference from the value immediately preceding iNO administration (i.e., value at $60 \mathrm{~min}$ ). 
a

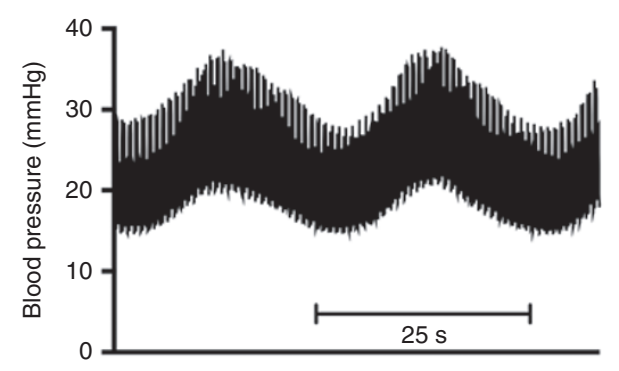

C

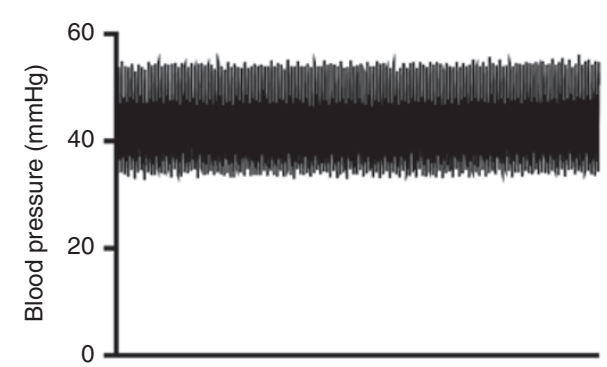

b

FGR

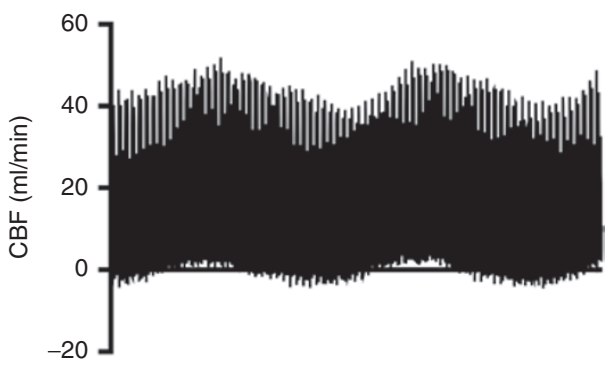

d

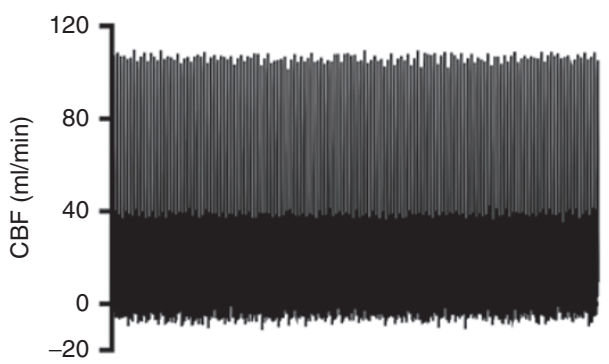

Figure 3. Loss of cerebral auto-regulation. Real time recording of carotid blood pressure (a and $\mathbf{c}$ ) and cerebral blood flow (CBF: $\mathbf{b}$ and $\mathbf{d})$ in a representative fetal growth restriction (FGR) (top panel) and appropriately grown (AG) preterm lamb taken $1 \mathrm{~h}$ after the initiation of ventilation. FGR lamb demonstrates harmonic mean pulse pressure variability and concurrent fluctuations in CBF. The lower blood pressure and flow indicates progressive cardiac failure in the FGR lamb while the AG lamb shows normal blood pressure and CBF.

or thromboxane mimetic (middle cerebral) between FGR and AG fetuses).

Third order femoral resistance arteries of FGR fetuses showed significantly impaired sensitivity $\left(\mathrm{pD}_{2}\right)$ and enhanced vasodilation to NO donor sodium nitroprusside (SNP) (Figure 4a) when compared with AG fetuses. Endothelial-dependent vasodilation to methacholine (MetCh) was significantly impaired (Figure 4b) in FGR fetuses compared with AG controls. Middle-cerebral arteries showed no difference in either endothelium-independent (SNP) or endothelium-dependent (MetCh) vasodilation (data not shown). Both third order femoral and middle-cerebral arteries demonstrated a significantly enhanced vasodilator response to sildenafil (Figure 4c, $\mathrm{d}$, respectively). In the middle-cerebral arteries this was also associated with impaired sensitivity.

\section{NO Expression and Distribution within the Lung}

To examine whether there were pre-existing deficiencies in NO related pathways in the lung, we examined lung tissue mRNA expression of endothelial nitric oxide synthase (eNOS), inducible nitric oxide synthase (iNOS) and vascular endothelial growth factor receptor (VEGFR-2), and protein expression and distribution of eNOS (Figure 5). There was a trend for decreased lung mRNA expression of VEGFR-2 and increased vascular endothelial growth factor (VEGF) expression in FGR lambs compared with AG lambs. Lung mRNA levels of eNOS were lower but did not reach significance due to the large variability in AG lambs. Protein expression of eNOS in lung tissue was not different between groups. However, histological examination of the distribution of eNOS showed reduced expression and distribution of eNOS in resistance arterioles within the lung of FGR lambs, with expression focused within endothelial cells (Figure 5e,f).

\section{Responsiveness to Inhaled NO Challenge}

After preliminary assessment of cardiovascular physiology and the demonstrated deficiencies in the NO pathway by the functional and histological analyses, we later investigated the physiological responsiveness to inhaled NO for $30 \mathrm{~min}$, in a subset ( $n=5$ each) of the ventilated groups.

Inhaled NO challenge to FGR lambs significantly increased mean and peak systolic PBF (Figure 2a,b) compared with immediately preceding iNO which coincided with a $\sim 25 \pm 3 \%$ decrease in PBF Pulsatility index (not significant). Mean PBF in FGR lambs was significantly higher throughout iNO administration than AG lambs while peak systolic PBF was higher from $75 \mathrm{~min}$. End diastolic PBF was not altered by iNO administration in either group (Figure 2c), and remained positive, indicative of pure left-to-right shunt across the ductus arteriosus in all lambs.

Mean, peak-systolic and end-diastolic CBF was not altered by iNO in FGR lambs (Figure 2d-f) but mean CBF was significantly reduced (by 50\%) in AG lambs while end diastolic $\mathrm{CBF}$ was significantly reduced at $80 \mathrm{~min}$. Pulsatility index measured in the carotid artery was not altered by iNO administration in FGR lambs, but significantly increased in AG lambs during iNO administration. Blood pressure (at 70 and $75 \mathrm{~min}$; Figure $2 \mathbf{h}$ ) and LVO (Figure 2i) was increased in FGR lambs during iNO administration while BP and LVO was not altered by iNO in AG lambs.

Upon cessation of iNO administration, mean and peak PBF, and blood pressure in FGR lambs returned to pre-iNO values. 


\section{Articles | Polglase et al.}

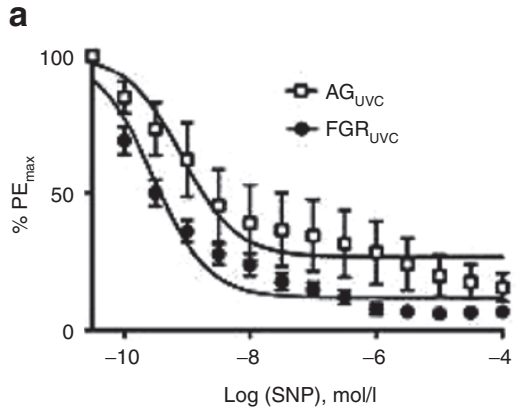

C

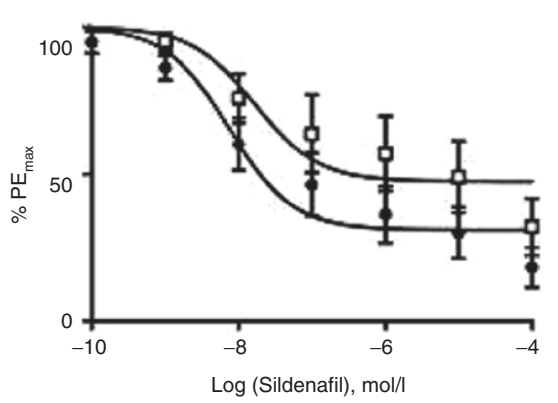

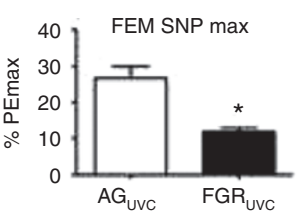
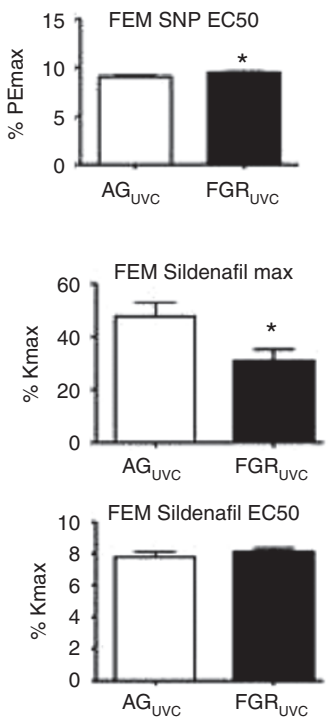

b
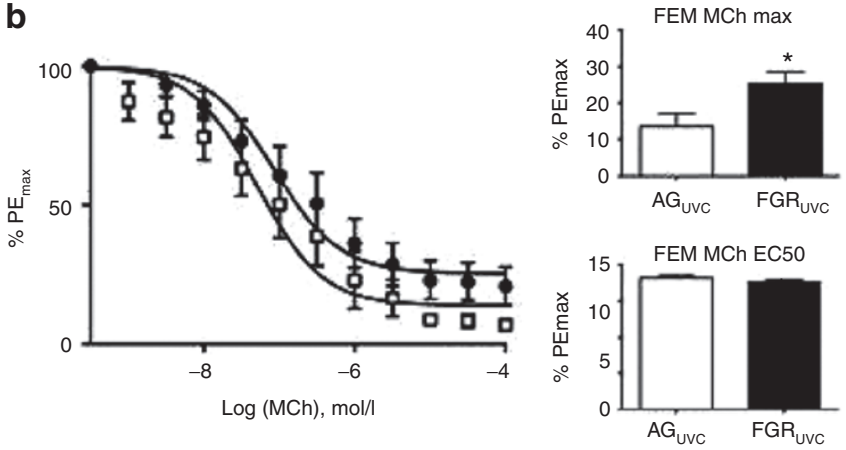

d

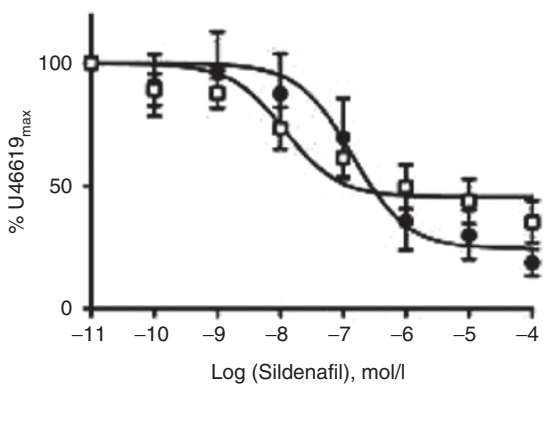

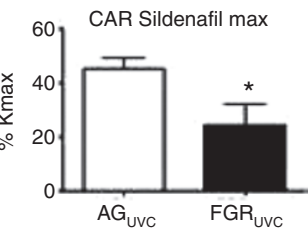

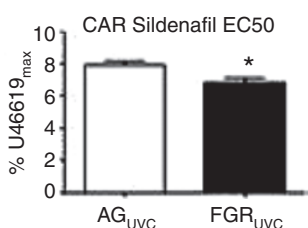

Figure 4. Vascular reactivity. Femoral artery (panels a-c) dose response curves to (a) sodium nitroprusside (SNP), (b) methylcholine (MCh), (c) sildenafil and (d) middle cerebral artery response to sildenafil in fetal growth restriction (FGR) (black circles) and appropriately grown (AG) (open squares) preterm lambs. Data are mean \pm SEM as a percentage of the maximal response to phenylephrine (\%PE Max, femoral arteries) and thromboxane (\%U46619 Max, middle cerebral arteries). Bar graphs show total maximal relaxation (Max) and the half maximal response (EC50) derived from line of best fit. * indicates significant difference $(P<0.05)$ between FGR and AG preterm lambs.

a

d

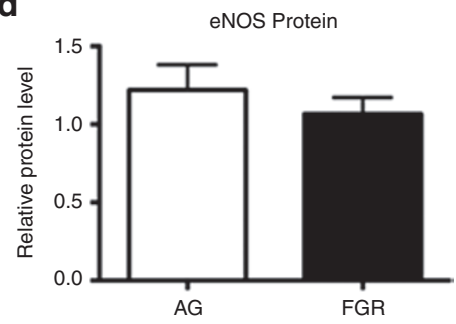

b

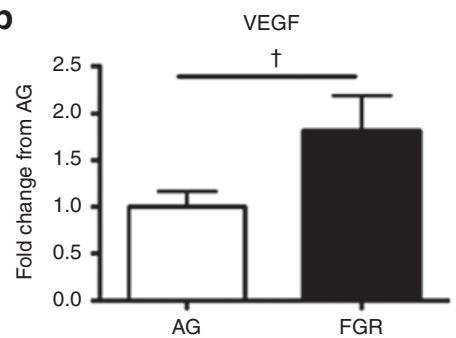

e

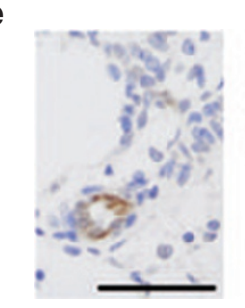

C

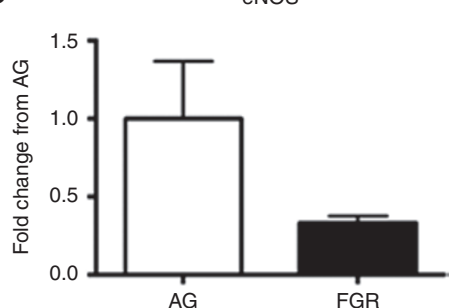

f

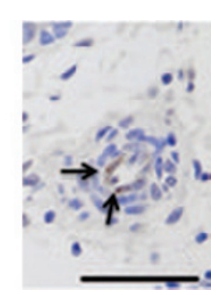

Figure 5. Nitric oxide (NO) pathway within the lung. Lung tissue mRNA expression of (a) vascular endothelial growth factor receptor 2 (VEGFR-2; also known as FLK-1), (b) all combined isotopes of vascular endothelial growth factor (VEGF), and (c) endothelial nitric oxide synthase (eNOS) in in fetal growth restriction (FGR) (black bars) and appropriately grown (AG) (white bars) preterm lambs. (d) Lung tissue protein expression levels of eNOS and localization of eNOS expression in resistance arterioles in (e) AG and (f) FGR preterm lambs; images taken at 400× magnification. Arrows indicate localized expression of eNOS to endothelial cells within the blood vessels of FGR lambs. † indicates trend $(P \leq 0.06)$ between FGR and AG preterm lambs. Scale bar is $50 \mu \mathrm{m}$.

Mean and end-diastolic CBF, and pulsatility index in the carotid artery returned to control values after cessation of iNO in AG lambs. Peak-systolic CBF in AG lambs continued to decrease and was significantly lower than pre-iNO values from $110 \mathrm{~min}$.

\section{DISCUSSION}

FGR imposes important and diverse adverse implications for life after birth. Growth restriction in utero is associated with long-term cardiovascular, pulmonary, and brain consequences 
that have been measured in children and adults $(1,5,16,17)$. We proposed that antenatal (fetal) adaptation to placental insufficiency and chronic hypoxia alter cardiovascular transition immediately after birth in FGR preterm offspring. Accordingly, we have studied the physiological, hemodynamic, and functional cardiovascular consequences of FGR in preterm newborn ventilated lambs. Our results demonstrate that the cardiovascular response in FGR preterm lambs is significantly different to AG lambs in the newborn transition period: FGR lambs had lower LVO and CBF; and higher systemic vascular resistance immediately after birth, and a trend to lower cerebral oxygenation, compared with AG preterm lambs. These physiological differences during the transition likely result from altered vascular function in FGR preterm lambs. We also identified inhibition of the NO pathway as a possible mechanism of altered cardiovascular function in FGR preterm lambs, evidenced by physiological, isolated vascular function, and histological analyses.

It is well established that FGR results in adaptations to cardiac function. The fetal response to suboptimal placental function includes a progressively increasing systemic afterload causing fetal myocardial dysfunction during systole and diastole, reduced contractile function, and reduced ventricular outputs $(2,18)$. Changes in placental impedance in placental insufficiency affect the fetal peripheral circulation. Fetuses demonstrate increased peripheral arterial resistance (via vasoconstriction) and reduced cerebral and coronary vascular resistance (via vasodilation). These fetal pathologies persist into adulthood, as evidenced by vascular dysfunction, increased blood pressure and carotid intima-media thickness in adults that were born growth restricted (19-21). In this lamb study, in addition to reduced cardiac output we also observed increased systemic arterial pulsatility index in FGR lambs, a measure of the resistance to blood flow distal to the site of measurement. These results in our model of preterm FGR lambs mirror fundamental cardiovascular differences observed in newborn FGR infants. Term-born FGR infants examined at day $2-5$ after birth had significantly reduced cardiac function (systolic and diastolic), reduced left ventricular output and impaired peripheral arterial biophysical properties, including increased arterial stiffness, vascular resistance and raised arterial pressure compared with age-matched healthy infants $(7,8)$. Initial low cardiac output and CBF in preterm infants increases the risk of intraventricular hemorrhage (22), and poor cardiac function increases susceptibility to ischemicreperfusion injury. Increased peripheral vascular resistance and vessel wall thickness lead to increased blood pressure in childhood, and increased cardiovascular risk in adulthood (20). Our findings indicate that these adaptations to cardiac function may occur very early in gestation with potential adverse outcomes in preterm and term FGR infants.

SUAL induced placental insufficiency, chronic hypoxia, and asymmetric FGR in fetal and newborn lambs, reflecting the clinical characteristics (13-15) and degree of fetal hypoxia (23) observed in moderate to severe human FGR. We have previously demonstrated that SUAL-induced FGR caused hemodynamic adaptations in the fetus, with altered cardiac output and redistribution of blood flow to the brain and heart, resulting in brain sparing $(24,25)$. However, the effects of placental insufficiency and FGR on transition physiology, postnatal cardiovascular function, and circulatory stability have not been previously studied, nor have they been investigated using other experimental models of FGR. They remain poorly understood in humans. We observed that FGR preterm lambs had lower ventricular output and cerebral blood flow immediately after birth. Both AG and FGR animals exhibited the expected rise in PBF at birth (11), the observed differences in LVO and CBF immediately after birth are therefore likely a direct effect on cardiovascular function and systemic vasculature rather than an effect driven by the pulmonary vasculature per se. Indeed the hemodynamic consequences of FGR occurred in the absence of any observable underlying respiratory complications or increased need for ventilation (26). Further, we have previously demonstrated that SUAL-induced FGR does not alter lung structural development compared with AG controls (27) and that FGR preterm lambs have similar respiratory requirements, lung inflammation, and injury relative to $A G$ preterm lambs (26).

We undertook isolated vascular myography studies to interrogate the mechanisms underlying the increased systemic vascular resistance. We found that femoral and middle cerebral resistance arteries of FGR lambs had increased responsiveness and sensitivity to sodium nitroprusside, a NO donor, indicating a potential mechanism for the vascular dysfunction occurs via alterations to the NO pathway. Previous studies support this contention $(28,29)$. FGR induced by maternal hyperthermia in lambs result in profound reductions in pulmonary vessel density, abnormal pulmonary arterial epithelial cell function in vitro and altered eNOS signaling (30). Other studies have also established that endothelium-dependent NO bioavailability is reduced in FGR (28). Using lung tissue we showed alterations to expression of key components of the NO pathway, although these differences failed to reach significance, likely due to combination of tissue and vascular components in the samples. Indeed, histological examination showed profound reductions in localized eNOS expression in resistance arterioles in FGR lambs compared with AG preterm lambs.

Given the findings of impaired vascular sensitivity to NO donors and the altered expression of key components of the NO pathway within the lung, we examined what would be the physiological effects, if we administered iNO to a subgroup of lambs. iNO administration to FGR preterm lambs resulted in a significant increase in PBF, blood pressure, and cardiac output. This suggests that the lower cardiac output was due to poor pulmonary venous return due to high pulmonary vascular resistance resultant from inhibited NO vasodilation. Indeed, pulsatility index in the left main pulmonary artery, a measure of pulmonary vascular resistance, was reduced (although not significantly) upon iNO administration, indicating a reduction in pulmonary vascular resistance. It follows therefore, that an alternate therapy for FGR preterm infants may be to enhance NO availability to the lung and/or systemic circulation. This contention is supported by our observations of a significantly 
enhanced vasodilator response to sildenafil, a phosphodiesterase inhibitor type 5 (PDE5), in third order femoral and middle-cerebral arteries from FGR lambs. Phosphodiesterase inhibitor (PDE) is responsible for breaking down cyclic guanosine-monophosphate that forms in response to increased NO. Inhibiting cyclic guanosine-monophosphate-dependent phosphodiesterase increases intracellular cyclic guanosinemonophosphate thus enhancing smooth muscle relaxation and vasodilation. Indeed, Sildenafil is currently used in infants with persistent pulmonary hypertension of the newborn and administration reverses pulmonary hypertension resulting in increased PBF, cardiac output and systemic perfusion $(31,32)$. Sildenafil is currently being examined as an antenatal therapy for early-onset human FGR (33). The importance of giving NO donors to the FGR preterm lung is further highlighted by the reduction in $\mathrm{PBF}$ and blood pressure, and restoration of high pulsatility index within the carotid artery upon cessation of iNO.

Our lamb study does however provide a cautionary note. We observed that cerebral blood flow was reduced by half in AG preterm lambs after iNO administration, suggesting that iNO may have detrimental hemodynamic consequences in AG preterm infants when it is not specifically indicated.

An interesting observation in this study was mechanical ventilation induced mean pulse pressure variability in arterial pressure in $44 \%$ of FGR preterm lambs, which was not observed in AG preterm lambs. Pulse pressure variability is known to increase with right ventricular failure (34) or interference with venous return to the left ventricle (35). Interestingly, concurrent fluctuations in carotid blood flow occurred with the fluctuations in arterial pressure, indicative of pressure-passivity and impaired cerebral auto-regulation. Human preterm infants show an association between low birth weight and pressure passivity similar to our observations (36). Failure of CBF to autoregulate is a causal factor leading to adverse pathologies including hypoxia/ischemia and intraventricular hemorrhage (37). In human infants, intraventricular hemorrhage occurred in 21 of 23 infants in which fluctuations in cerebral blood flow velocity (measured by Doppler ultrasound) were detected (38). The cause of the impaired cerebral autoregulation in FGR preterm lambs is not known, but likely result from adverse cerebral vascular development or function, or impaired cardiovascular control.

\section{Conclusion}

This study demonstrates that the fetal circulatory adaptation to hypoxia alters the cardiovascular physiology of preterm FGR infants in the immediate newborn period compared with AG preterm infants. This has important clinical implications, as FGR infants will respond differently to clinical interventions, particularly those aimed at improving cardiovascular stability. The maintenance of low cardiac output and high vascular resistance in our preterm FGR lambs may be causal factors leading to enhanced neonatal complications observed in growth restricted infants, with the underlying mechanism likely impaired NO sensitivity - a potential therapeutic target. This study clearly shows that FGR preterm infants are a distinctly different cohort of preterm neonates. We propose that individualized care is warranted for preterm FGR infants, targeting improved cardiovascular output and vascular function in the first days of life.

\section{METHODS}

\section{Surgical Preparation}

Twin bearing Border-Leicester ewes of known mating date underwent aseptic surgery at $103-105$ d gestation (term is $\sim 148 \mathrm{~d}$ ) to induce FGR by SUAL in one twin, using the other twin as a control as described previously $(24,39)$. Briefly, anesthesia was induced via an injection of sodium thiopentane ( $20 \mathrm{ml}$; Pentothal; Jurox, Rutherford, Australia) and maintained after intubation with a cuffed endotracheal tube (ID $8.0 \mathrm{~mm}$, OD $10.9 \mathrm{~mm}$, Portex, England) with Isoflurane $(1.5-2.5 \%$ in $100 \%$ oxygen). Antibiotics; Ampicillin (Jurox), $1 \mathrm{~g}$ intravenous, and Engemysin (Coopers Animal Health, Bendigo East, Australia) $5 \mathrm{ml}$, intravenous, were administered to the ewe immediately following the induction of anesthesia, and were maintained for $3 \mathrm{~d}$ post surgery. A hysterectomy was performed and each fetus was exposed and a catheter placed in a femoral artery for monitoring of fetal wellbeing. In one fetus, two ligatures were tied around one umbilical artery (SUAL), while the cord was manipulated in the control fetus without ligation. Following instrumentation, the fetuses were returned to the uterus, and the uterus and abdominal incisions sites were repaired. Fetal wellbeing was monitored every second day by assessing bloodgas and electrolyte status (ABL 700 blood-gas analyzer; Radiometer, Copenhagen, Denmark).

\section{Delivery and Ventilation of Preterm Lambs}

At $125 \mathrm{~d}$ gestation, the ewe and fetus were anaesthetized as above, and the fetal head and chest were exposed via hysterectomy for placement of a 4-mm ultrasonic flow transducer (Transonic Systems, Ithaca, $\mathrm{NY}$ ) around the left main pulmonary artery. A flow-probe (3-mm) was placed around the carotid artery, and catheters were inserted into a brachial artery and femoral vein. Arterial pressures and blood flows were digitally recorded in real-time $(1 \mathrm{kHz}$, Powerlab; ADInstruments, Castle Hill, NSW, Australia). After closure of the incisions in the neck and chest, the fetal trachea was intubated with a $4.0 \mathrm{~mm}$ cuffed endotracheal tube and lung liquid was drained passively. A transcutaneous arterial oxygen saturation $\left(\mathrm{SpO}_{2}\right)$ probe (Masimo, Radical 4, CA) was placed around the right forelimb of the lamb and the output digitally recorded. A Near Infrared Spectroscopy optode (Casmed Foresight, CAS Medical Systems, Branford, CT) was placed over the left frontal cortex and used to continuously measure cerebral tissue oxygen saturation $\left(\mathrm{SctO}_{2}\right)$.

The umbilical cord was then clamped and cut, the lambs were delivered, dried, weighed and placed on an infant warmer (Fisher and Paykel Healthcare, Auckland, New Zealand) for initiation of ventilation. An umbilical vein was immediately catheterized for maintenance of anesthesia and analgesia (Alfaxane intravenous $5 \mathrm{mg} / \mathrm{kg} / \mathrm{h}$; Jurox). The lambs were anaesthetized for the entirety of the experiment to prevent spontaneous breathing. Ventilation was commenced using positive pressure ventilation with peak inflation pressure (PIP) set at $40 \mathrm{~cm} \mathrm{H}_{2} \mathrm{O}$ and positive end-expiratory pressure (PEEP) at $5 \mathrm{~cm}$ $\mathrm{H}_{2} \mathrm{O}$ (Babylog 8000+, Dräger, Lübeck, Germany): inspiratory time was $0.4 \mathrm{~s}$ and expiratory time was $0.6 \mathrm{~s}$. Lambs were ventilated with warmed, humidified gas with an initial fraction of inspired oxygen $\left(\mathrm{F}_{\mathrm{i}} \mathrm{O}_{2}\right)$ of 0.4 and subsequently adjusted to maintain $\mathrm{SaO}_{2}$ between 90-95\%. At $10 \mathrm{~min}$, all lambs received surfactant (Curosurf, $100 \mathrm{mg}$ / $\mathrm{kg}$, Chiesi Farmaceutica, Palermo, Italy). At $20 \mathrm{~min}$, ventilation continued in volume guarantee mode set at $7 \mathrm{ml} / \mathrm{kg}$, which is the tidal volume for lambs at this gestation (40). Physiological parameters $\mathrm{pH}$ (7.2-7.4) and $\mathrm{PaCO}_{2}(45-55 \mathrm{mmHg}$ ) were kept within normal limits by adjusting the ventilator rate and inspired $\mathrm{O}_{2}$ levels. Lamb wellbeing was monitored throughout ventilation with regular blood-gas samples. 


\section{Doppler Echocardiography and Waveform Measurements}

Left ventricular output was detected using pulsed Doppler ultrasound as described previously (41). Real-time recordings of blood flow were captured via indwelling transonic flow probes immediately prior to delivery (fetal values) and throughout the $2 \mathrm{~h}$ ventilation strategy. Transonic flow probes have accuracy within $1 \mathrm{ml} / \mathrm{min}$ and are considered the gold-standard for accurate and continuous measure of blood flow (42). Thirty s representative samples at each time point were collected and analyzed for mean, peak-systolic and end-diastolic flows using data acquisition and analysis software (LabChart v7.2.5, ADInstruments, Sydney, Australia) as described previously (43).

\section{Vasodilator Challenge}

After initial analysis of findings suggested a deficiency in NO signaling, we explored the therapeutic potential of iNO in a subgroup of ventilated preterm AG and FGR lambs ( $n=5$ each). The methodology was the same as above, however at $60 \mathrm{~min}$ during ventilation, lambs received iNO, $20 \mathrm{ppm}$; (Ikaria, iNOVENT, NJ) via the endotracheal tube for $30 \mathrm{~min}$. At $90 \mathrm{~min}$, iNO was stopped, and the lambs remained under ventilator support until $120 \mathrm{~min}$. At the conclusion of the experiment, ewes and lambs were humanely killed (sodium pentobarbitone, Valabarb, Jurox; $>100 \mathrm{mg} / \mathrm{kg}$ intravenous). Lung and blood vessels were collected and examined as described below.

\section{Vascular Reactivity}

An additional cohort of AG and FGR fetuses ( $n=6$ for both) underwent the initial surgery however were delivered and euthanased immediately at $125 \mathrm{~d}$ gestation, prior to ventilation (unventilated controls: $\mathrm{AG}_{\mathrm{UVC}}$ and $\mathrm{FGR}_{\mathrm{UVC}}$ respectively). In these fetuses, third order femoral and middle cerebral arteries were located, isolated and carefully removed for in vitro wire myography.

Femoral and middle-cerebral arteries were mounted on a fourchamber wire myograph (Multi Wire Myograph System 610M, DMT, Denmark) containing Krebs solution. Throughout experimentation the Krebs solution was changed every $15 \mathrm{~min}$, with $>20$ min between experiments to allow vessel reactivity to return to baseline. Following normalization (to $90 \%$ of $5.3 \mathrm{kPA}$; DMT normalization module), endothelial vascular reactivity was confirmed. Vasoconstriction response curves were gained for high $\mathrm{K}+$ physiological saline solution, phenylephrine (PE; concentration range $10^{-9}-10^{-4} \mathrm{~mol} / \mathrm{l}$; Sigma P6126, Sigma-Aldrich, Castle Hill, Australia) and thromboxane (U46619; concentration range $10^{-10}-10^{-6} \mathrm{~mol} / \mathrm{l}$ ) in the femoral and middle-cerebral arteries, respectively. Vasodilation response curves in response to SNP; concentration range $10^{-10}-10^{-4} \mathrm{~mol} / \mathrm{l}$; (Sigma 71778, Sigma-Aldrich), methacholine (MetCh; concentration range $10^{-10}-10^{-4} \mathrm{~mol} / \mathrm{l}$; Sigma A2251) and sildenafil (concentration range $10^{-10}-10^{-4} \mathrm{~mol} / \mathrm{l}$; Sigma PZ0003, Sigma-Aldrich) were determined after submaximal precontraction with the relevant vasoconstrictor (femoral: PE, middle-cerebral: U46619). To determine the contribution of NO to endothelial-dependent vasodilation a final vasodilation response curve to $\mathrm{MCh}$ was also conducted in the presence of NO inhibitor L-NAME $\left(10^{-5} \mathrm{~mol} / \mathrm{l}\right.$; Sigma N5751, Sigma-Aldrich). Concentration-response curves were analyzed using an agonistresponse line of best fit. The maximal relaxant response (\% Rmax) was expressed as percentage of the submaximal precontraction and vascular sensitivity was expressed as $\mathrm{pD}_{2}\left(-\log ^{\mathrm{EC} 50}\right)$. NO-dependent vasodilation was calculated by subtracting the area under the curve for MetCh from the area under the curve for MetCh + L-NAME. The EC50, which is the concentration of agonist that provokes a response halfway between the baseline and maximum response of the vasodilation curve was calculated (Prism 6, GraphPad Software, La Jolla, CA).

\section{Measurements and Calculations}

Dynamic compliance $\left(\mathrm{C}_{\mathrm{dvp}}\right)$, adjusted for bodyweight, was calculated as $\mathrm{V}_{\mathrm{T}} / \mathrm{kg} /(\mathrm{PIP}-\mathrm{PEEP})$. Ventilatory efficiency index was calculated as $3800 /\left(\Delta P \cdot f \cdot P \mathrm{aCO}_{2}\right)$ where 3800 is a carbon dioxide production constant $\left(\mathrm{ml} \cdot \mathrm{mmHg} \cdot \mathrm{kg}^{-1} \cdot \mathrm{min}^{-1}\right), \Delta P=$ PIP-PEEP and $f$ is the respiratory frequency. The alveolar-arterial difference in oxygen $\left(\mathrm{AaDO}_{2}\right)$ was calculated as $\mathrm{AaDO}_{2}=\left(\mathrm{FiO}_{2} \times(760-47 \mathrm{mmHg})-\mathrm{PaCO}_{2} / \mathrm{RQ}\right)-\mathrm{PaO}_{2}$, where RQ is the respiratory quotient, which is $\sim 0.93$ in newborn lambs. Pulsatility index was determined from samples of the waveforms from the left main pulmonary artery and left carotid artery by: (peak systolic blood flow - end diastolic blood flow)/mean blood flow.

\section{Molecular Assessment of the NO Pathway}

The lungs were dissected and the right lower lobe was snap frozen and RNA was extracted for quantitative real-time PCR. Snap frozen tissue was homogenized and total RNA was isolated (RNeasy Maxi Kit, Qiagen, Chadstone, Australia) and reverse-transcribed into cDNA (SuperScript III reverse transcriptase, Invitrogen; Life Technologies, Australia, Scoresby, Australia). Relative mRNA expression of eNOS, $i N O S$, and VEGF-R2 (otherwise known as Flk-1) genes were measured by quantitative real-time PCR using Applied Biosystems 7900 HT Fast Real-Time PCR system. The expression of all genes was normalized to the $18 \mathrm{~S}$ rRNA for each sample using the cycle threshold $\left(\Delta \mathrm{C}_{\mathrm{T}}\right)$ method of analysis and was expressed relative to the $\mathrm{Sal}_{\mathrm{UVC}}$ or LPS $_{\text {UVC }}$ group.

\section{Western Blot Analysis}

Western blot analysis was performed using $50 \mu \mathrm{g}$ of lung protein with a monoclonal antibody to eNOS (BD Biosciences, Sydney, Australia). Multiple blots were required to analyze the large number of animals studied. To ensure comparable transfer conditions between gels, one sample was chosen to run on both gels as an internal control. Image of the membranes were captured by ChemiDoc XRS system (BIO-Rad Laboratories, Gladesville, Australia) and analyzed using ImageJ (National Institutes of Health, Bethesda, MD). eNOS level is expressed as density relative to beta-actin density normalized to the internal control sample.

\section{Immunohistochemistry Staining for eNOS Protein}

The lungs were pressure fixed with $10 \%$ buffered Formalin and embedded in paraffin, then cut into $5 \mu \mathrm{m}$ section and mounted on to Superfrost Plus slides. For immunostaining, slides were heated in $60{ }^{\circ} \mathrm{C}$ oven for $2 \mathrm{~h}$ to melt away excessive wax, followed with histolene clearing and ethanol rehydrating steps. Antigen retrieval was performed using $0.01 \mathrm{~mol} / \mathrm{l}$ Citrate buffer ( $\mathrm{pH}$ 6.0). Endogenous peroxidase in the tissue was blocked with $3 \%$ hydrogen peroxide for $10 \mathrm{~min}$. The slides were then blocked with $5 \%$ bovine serum albumin with $0.5 \%$ triton $\mathrm{X}$ before incubate with eNOS monoclonal antibody (BD Biosciences) at 1:100 dilution overnight at $4{ }^{\circ} \mathrm{C}$. Sections then incubated with biotinylated anti-mouse secondary (1:200) followed with streptavidin horseradish peroxidase and developed with diaminobenzidine and hydrogen peroxide. Sections were counterstained with hematoxylin and dehydrated with ethanol and histolene before mounting with coverslip.

\section{Statistics}

Serial samples collected from the fetus were compared using two-way repeated measures ANOVA with post-hoc analysis (Holm-Sidak) determining the time that differences were evident (Sigmastat v3.0, SPSS, San Jose, CA). Cord blood gas data collected immediately prior to intervention were compared using Students $t$-test. Average values were obtained from recordings of $10 \mathrm{~s}$ duration during the first $10 \mathrm{~min}$ after birth and for the first $10 \mathrm{~min}$ after vasodilator challenge. For all other time points the average values were obtained from 30 -s recordings. Serial data were compared over time and between groups using two-way repeated measures ANOVA for postnatal physiological data with post-hoc analysis (Holm-Sidak) determining the time that differences became evident. Changes in hemodynamic indices during and immediately after iNO challenge were compared with the value immediately preceding iNO challenge $(60 \mathrm{~min})$ using two-way repeated measures ANOVA and post-hoc analysis as above. Data from in vitro wire myography was compared using $t$-test. Data are presented as mean \pm SEM unless otherwise stated. Statistical significance was accepted for $P<0.05$.

\section{Ethics Statement}

The experimental protocol was performed in accordance with guidelines established by the National Health and Medical Research Council of Australia and received approval of the Monash Medical Centre, Monash University animal ethics committee.

\section{ACKNOWLEDGMENTS}

We would like to acknowledge the assistance of Valerie Zahra, Alison Moxham, Karyn Rodgers, Ilias Nitsos, Jan Loose, Tamara Yawno, and Amy Sutherland who assisted with the animal studies. 


\section{STATEMENT OF FINANCIAL SUPPORT}

This research was supported by a National Health and Medical Research Council (NH\&MRC; Canberra, Australia) Project Grant (APP1083520), a grant from the Arthur \& Mary Osborn Charitable Trust and the Hugh D. T. Williamson Foundation, which are managed by Equity Trustees (Melbourne, Australia), NH\&MRC Research Fellowships (GRP: 1026890 and SBH: 545921), an Australian Research Council Future Fellowship (SLM; Canberra, Australia), a Rebecca L. Cooper Medical Research Foundation Fellowship (GRP; Edgecliffe, Australia) and the Victorian Government's Operational Infrastructure Support Program.

Disclosure: Ikaria Australia generously donated the iNOVENT and gas used for the pulmonary vasodilator challenge. They had no role in the design of the project or the interpretation of the findings.

\section{REFERENCES}

1. Rosenberg A. The IUGR newborn. Semin Perinatol 2008;32:219-24.

2. Baschat AA. Pathophysiology of fetal growth restriction: implications for diagnosis and surveillance. Obstet Gynecol Surv 2004;59:617-27.

3. Resnik R. Intrauterine growth restriction. Obstet Gynecol 2002;99:490-6.

4. Piper JM, Xenakis EM, McFarland M, Elliott BD, Berkus MD, Langer O. Do growth-retarded premature infants have different rates of perinatal morbidity and mortality than appropriately grown premature infants? Obstet Gynecol 1996;87:169-74.

5. Pallotto EK, Kilbride HW. Perinatal outcome and later implications of intrauterine growth restriction. Clin Obstet Gynecol 2006;49:257-69.

6. McMillen IC, Adams MB, Ross JT, et al. Fetal growth restriction: adaptations and consequences. Reprod 2001;122:195-204.

7. Sehgal A, Doctor T, Menahem S Cardiac function and arterial indices in infants born small for gestational age: analysis by speckle tracking Acta Paediatr 2013;15:12465.

8. Sehgal A, Doctor T, Menahem S. Cardiac function and arterial biophysical properties in small for gestational age infants: postnatal manifestations of fetal programming. J Pediatr 2013;163:1296-300.

9. Rudolph AM. Fetal and neonatal pulmonary circulation. Annu Rev Physiol 1979;41:383-95.

10. Hooper SB, Harding R Role of aeration in the physiological adaptation of the lung to air-breathing at birth. Cur Resp Med Rev. 2005;1:185-195.

11. Bhatt $\mathrm{S}$, Alison BJ, Wallace EM, et al. Delaying cord clamping until ventilation onset improves cardiovascular function at birth in preterm lambs. J Physiol 2013;591:2113-26.

12. te Pas AB, Davis PG, Hooper SB, Morley CJ. From liquid to air: breathing after birth. J Pediatr 2008;152:607-11.

13. Miller SL, Supramaniam VG, Jenkin G, Walker DW, Wallace EM. Cardiovascular responses to maternal betamethasone administration in the intrauterine growth-restricted ovine fetus. Am J Obstet Gynecol 2009;201:613. e1-8.

14. Supramaniam VG, Jenkin G, Loose J, Wallace EM, Miller SL. Chronic fetal hypoxia increases activin A concentrations in the late-pregnant sheep. BJOG 2006;113:102-9.

15. Miller SL, Sutherland AE, Supramaniam VG, Walker DW, Jenkin G, Wallace EM. Antenatal glucocorticoids reduce growth in appropriately grown and growth-restricted ovine fetuses in a sex-specific manner. Reprod Fertil Dev 2012;24:753-8.

16. Barker DJ. Adult consequences of fetal growth restriction. Clin Obstet Gynecol 2006;49:270-83.

17. Fall CH, Vijayakumar M, Barker DJ, Osmond C, Duggleby S. Weight in infancy and prevalence of coronary heart disease in adult life. BMJ 1995;310:17-9.

18. Bahtiyar MO, Copel JA. Cardiac changes in the intrauterine growthrestricted fetus. Semin Perinatol 2008;32:190-3.

19. Cosmi E, Fanelli T, Visentin S, Trevisanuto D, Zanardo V. Consequences in infants that were intrauterine growth restricted. J Pregnancy 2011;2011:364-81.

20. Demicheva E, Crispi F Long-term follow-up of intrauterine growth restriction: Cardiovascular disorders Fetal Diagn Ther 2014;36:143-53.

21. Siddiqui N, Hladunewich M. Understanding the link between the placenta and future cardiovascular disease. Trends Cardiovasc Med 2011;21:188-93.
22. Kluckow M, Evans N. Low superior vena cava flow and intraventricular haemorrhage in preterm infants Arch Dis Child Fetal Neonatal Ed 2000;82:F188-194.

23. Soothill PW, Nicolaides KH, Campbell S. Prenatal asphyxia, hyperlacticaemia, hypoglycaemia, and erythroblastosis in growth retarded fetuses $\mathrm{Br}$ Med J (Clin Res Ed). 1987;294:1051-53.

24. Miller SL, Loose JM, Jenkin G, Wallace EM. The effects of sildenafil citrate (Viagra) on uterine blood flow and well being in the intrauterine growthrestricted fetus. Am J Obstet Gynecol 2009;200:102.e1-7.

25. Parkington HC, Tare M, Sutherland A, Jenkin G, Miller SJ, Wallace EM. Maternal betamethasone has significant adverse outcomes for heart function in growth restricted sheep fetuses. J Paediatr Child Health 2009;45:A57.

26. Allison BJ, Hooper SB, Coia E, et al. Ventilation-induced lung injury is not exacerbated by growth restriction in preterm lambs. Am J Physiol Lung Cell Mol Physiol 2016;310:L213-23.

27. Sutherland AE, Crossley KJ, Allison BJ, Jenkin G, Wallace EM, Miller SL. The effects of intrauterine growth restriction and antenatal glucocorticoids on ovine fetal lung development. Pediatr Res 2012;71:689-96.

28. Tare M, Parkington HC, Wallace EM, et al. Maternal melatonin administration mitigates coronary stiffness and endothelial dysfunction, and improves heart resilience to insult in growth restricted lambs. J Physiol 2014;592:2695-709.

29. Kane AD, Herrera EA, Camm EJ, Giussani DA. Vitamin C prevents intrauterine programming of in vivo cardiovascular dysfunction in the rat. Circ J 2013;77:2604-11.

30. Rozance PJ, Seedorf GJ, Brown A, et al. Intrauterine growth restriction decreases pulmonary alveolar and vessel growth and causes pulmonary artery endothelial cell dysfunction in vitro in fetal sheep. Am J Physiol Lung Cell Mol Physiol 2011;301:L860-71.

31. Abman SH. Recent advances in the pathogenesis and treatment of persistent pulmonary hypertension of the newborn. Neonatol 2007;91:283-90.

32. Herrera EA, Ebensperger G, Krause BJ, et al. Sildenafil reverses hypoxic pulmonary hypertension in highland and lowland newborn sheep. Pediatr Res 2008;63:169-75.

33. Ganzevoort W, Alfirevic Z, von Dadelszen P, et al. STRIDER: Sildenafil therapy in dismal prognosis early-onset intrauterine growth restriction-a protocol for a systematic review with individual participant data and aggregate data meta-analysis and trial sequential analysis. Syst Rev 2014;3:23.

34. Jardin F. Cyclic changes in arterial pressure during mechanical ventilation. Intensive Care Med 2004;30:1047-50.

35. Khasnis A, Lokhandwala Y. Clinical signs in medicine: pulsus paradoxus. J Postgrad Med 2002;48:46-9.

36. Soul JS, Hammer PE, Tsuji M, et al. Fluctuating pressure-passivity is common in the cerebral circulation of sick premature infants. Pediatr Res 2007;61:467-73.

37. Khwaja O, Volpe JJ. Pathogenesis of cerebral white matter injury of prematurity. Arch Dis Child Fetal Neonatal Ed 2008;93:F153-161.

38. Perlman JM, McMenamin JB, Volpe JJ. Fluctuating cerebral blood-flow velocity in respiratory-distress syndrome. Relation to the development of intraventricular hemorrhage. N Engl J Med 1983;309:204-9.

39. Miller SL, Chai M, Loose J, et al. The effects of maternal betamethasone administration on the intrauterine growth-restricted fetus. Endocrinol 2007;148:1288-95.

40. Polglase GR, Hillman NH, Ball MK, et al. Lung and systemic inflammation in preterm lambs on continuous positive airway pressure or conventional ventilation. Pediatr Res 2009;65:67-71.

41. Polglase GR, Hooper SB, Gill AW, et al. Cardiovascular and pulmonary consequences of airway recruitment in preterm lambs. J Appl Physiol (1985) 2009;106:1347-55.

42. Dean DA, Jia CX, Cabreriza SE, et al. Validation study of a new transit time ultrasonic flow probe for continuous great vessel measurements. ASAIO J 1996;42:M671-6.

43. Polglase GR, Morley CJ, Crossley KJ, et al. Positive end-expiratory pressure differentially alters pulmonary hemodynamics and oxygenation in ventilated, very premature lambs. J Appl Physiol (1985) 2005;99: 1453-61. 